\title{
A VISÃo RELACIONAL DE POLÍTICAS DE CIÊNCIA E TECNOLOGIA: O CASO DO PROGRAMA CI BRASIL
}

\author{
Kadígia Faccin* \\ kadigia@gmail.com \\ Ingridi Bortolaso ** \\ ingridibortolaso@yahoo.com.br
}

\author{
Alsones Balestrin* \\ abalestrin@unisinos.br
}

*Universidade do Vale do Rio dos Sinos - São Leopoldo, RS / Brasil

**Universidade de Santa Cruz do Sul - Santa Cruz do Sul, RS / Brasil

http://dx.doi.org/10.1590/1413-2311.070142014.54831

Recebido em 13/04/2015

Aprovado em 24/05/2016

Disponibilizado em 06/06/2016

Avaliado pelo sistema "double blind review"

Revista Eletrônica de Administração

Editora-chefe: Aurora Zen

ISSN 1413-2311 (versão "on line")

Editada pela Escola de Administração da Universidade Federal do Rio Grande do Sul.

Periodicidade: Quadrimestral

Sistema requerido: Adobe Acrobat Reader

\section{RESUMO}

A literatura sobre cooperação interorganizacional tem trazido ao debate acadêmico, especialmente das últimas duas décadas, uma reflexão sobre os resultados da atuação conjunta de diversos atores para alcançar fins comuns. Essa forma de pensar os meios para alcançar os fins pretendidos foi denominada por Dyer e Singh (1998) como "visão relacional". De acordo com a visão relacional, a estratégia coletiva cria uma fonte inimitável de recursos através de uma rede com acesso valioso a informações, compartilhamento de conhecimento, complementaridade de recursos e governança. $\mathrm{O}$ presente estudo visa ao entendimento sobre como os Governos utilizam a visão relacional para desenvolver com maior eficácia suas políticas públicas de ciência e tecnologia. A estratégia de pesquisa utilizada foi o estudo de caso e o objeto empírico analisado foi o Programa Governamental de Circuitos Integrados CI Brasil, que foi criado com o objetivo de desenvolver recursos humanos qualificados à indústria brasileira de semicondutores. O objetivo do artigo é trazer evidências sobre como uma política pública, em uma área complexa de ciência e tecnologia, pode ser colocada em prática por meio de uma ação articulada entre diversos atores públicos e privados. $\mathrm{O}$ artigo enfatiza a importância das relações recíprocas entre Universidade, Indústria e Governo para a eficácia na implementação de políticas públicas.

Palavras-Chave: Visão Relacional; Política de Ciência e Tecnologia; Programa CI-Brasil. 


\title{
A VISÃO RELACIONAL DE POLÍTICAS DE CIÊNCIA E TECNOLOGIA: O CASO DO PROGRAMA CI BRASIL
}

\section{THE RELATIONAL VIEW ON SCIENCE POLICY AND TECHNOLOGY: THE CASE OF THE CI BRAZIL PROGRAM}

\begin{abstract}
The literature point of view on interorganizational cooperation has brought to the academic debate, especially in the last two decades, a reflection on the results of the joint efforts of various actors to achieve common goals. This way of thinking of the means to achieve its intended purpose was named by Dyer and Singh (1998) "relational view". According to the relational view, the collective strategy creates an inimitable source of resources through a network with access to information, knowledge sharing, complementarity of resources and governance. This study aims at understanding how governments use the relational view in order to more effectively develop their public policies science and technology. The research strategy used was the case study. The empirical object of study is the CI Brazil Governmental Program, which was created with the goal of developing the semiconductor industry in Brazil. The paper presents evidence on how public policy, in a complex area of science and technology, can be put into practice in an emerging country. The article shows the importance of reciprocal relationships between university, industry and government to make the implementation of the public policy happen.
\end{abstract}

Keywords: Relational View; Policy of Science and Technology; CI Brazil Program.

\section{LA VISIÓN RELACIONAL DE LAS POLÍTICAS DE CIENCIA Y TECNOLOGÍA: EL CASO DEL PROGRAMA CI BRASIL}

\begin{abstract}
RESUMEN
La literatura sobre cooperación interorganizacional ha traído al debate académico, especialmente de las últimas dos décadas, una reflexión sobre los resultados de la actuación conjunta de diversos actores para alcanzar fines comunes. Esa forma de pensar los medios para alcanzar los fines pretendidos fue denominada por Dyer y Singh (1998) de "visión relacional". Según la visión relacional, la estrategia colectiva crea una fuente inimitable de recursos a través de una red con acceso valioso a informaciones, compartimiento de conocimiento, complementariedad de recursos y gobernanza. El presente estudio tiene como objetivo la comprensión de como los gobiernos utilizan la visión relacional para desarrollar con máxima eficacia sus políticas públicas de desarrollo industrial . La estrategia de búsqueda utilizada fue caso de estudio. El objetivo empírico del estudio es el programa gubernamental CI Brasil que fue creado con la meta de desenvolver la industria de semiconductores en Brasil. El artículo presenta evidencias sobre como una política pública, en un área compleja de ciencia y tecnología, puede ser colocada en práctica en un país emergente. El artículo destaca la importancia de las relaciones recíprocas entre universidad, industria y gobierno para la aplicación eficaz de la política pública.
\end{abstract}

Palabras Clave: Visión Relacional; Política de Ciencia y Tecnología; Programa Brasileño Ci - Brasil. 


\section{Kadígia Faccin, Ingridi Bortolaso \& Alsones Balestrin}

\section{INTRODUÇÃO}

A literatura sobre cooperação interorganizacional tem trazido ao debate acadêmico, especialmente das últimas duas décadas, uma reflexão sobre os resultados da atuação conjunta de diversos atores para alcançar fins comuns. Essa forma de pensar os meios para alcançar os fins pretendidos foi denominada por Dyer e Singh (1998) como “visão relacional”. De acordo com a visão relacional, a estratégia coletiva cria uma fonte inimitável de recursos através de uma rede de relacionamentos com acesso valioso a informações, compartilhamento de conhecimento, complementaridade de recursos e governança conjunta. Os autores da visão relacional da estratégia têm utilizado como foco empírico, na maior parte de seus trabalhos, a busca do entendimento dos ganhos relacionais percebidos pela atuação conjunta de empresas ou organizações em diversos tipos de indústrias.

Por outro lado, as transformações recentes da economia mundial tornaram o desenvolvimento nacional em ciência, tecnologia e inovação uma das estratégias para aumentar a produção, melhorar a qualidade de vida da população e enfrentar os problemas sociais, urbanos e ambientais. De acordo com Schwartzman (1993), as políticas de ciência e tecnologia são proveitosas quando associadas a políticas coerentes de ajuste econômico e de desenvolvimento industrial e educacional. Além disso, “[...] as políticas do Governo Federal só podem ser efetivas quando combinadas com a participação ativa dos Governos Estaduais e locais e de setores sociais significativos, como o empresariado, os trabalhadores, educadores e cientistas" (SCHWARTZMAN, 1993, p. 8).

Nesse contexto, uma problemática que necessita ser aprofundada é o entendimento de como os Governos utilizam estratégias relacionais para desenvolver com maior eficácia suas políticas públicas de desenvolvimento industrial. A ação conjunta entre Universidade, Indústria e Governo U-I-G, por exemplo, tem se tornado uma real possibilidade de ganho de sinergia e complementaridade para que políticas públicas obtenham melhores resultados (SANDFORT; MILWARD, 2008).

Em países em desenvolvimento, como é o caso do Brasil, a colaboração entre esses atores torna-se ainda mais imperativa, pois a sinergia coletiva em ações articuladas com diversos atores poderá atenuar os recursos escassos. Entre os diversos exemplos de ações conjuntas entre U-I-G, especialmente na de ciência e tecnologia, uma em especial tem gerado importantes resultados junto a indústria brasileira de semicondutores. O Governo Brasileiro implantou, em 2004, uma política pública denominada "Programa CI Brasil", que visa ao desenvolvimento de um ecossistema sustentável para o desenvolvimento da indústria nacional 


\section{A VISÃO RELACIONAL DE POLÍTICAS DE CIÊNCIA E TECNOLOGIA: O CASO DO PROGRAMA CI BRASIL}

de semicondutores. A sigla CI refere-se à "Circuito Integrado" um dos principais componentes de equipamentos eletrônicos. Assim, de forma colaborativa, U-I-G atuam para a implementação da política pública proposta e compartilham o objetivo de fazer com que o Brasil tenha uma indústria consolidada para o setor.

Diante desse contexto teórico-empírico, a presente pesquisa tem como objetivo descrever os antecedentes e os resultados alcançados pelo Programa CI Brasil, bem como a dinâmica relacional de colaboração entre os seus três atores institucionais - U-I-G. Para atingir esse objetivo, foi conduzido um estudo de caso do Programa CI Brasil, com técnicas de coleta de dados por meio de entrevistas e análise documental. As entrevistas foram realizadas com especialistas, profissionais e pesquisadores vinculados ao Programa, escolhidos pelo critério de conhecimento e experiência na indústria de semicondutores e, em especial, junto ao caso em análise.

Com vista a alcançar o objetivo proposto, o artigo está estruturado da seguinte forma: na primeira parte, apresenta-se o referencial teórico que embasou a pesquisa; na segunda, é apresentado a metolodogia de pesquisa. Na sequência, apresenta-se a análise dos resultados e, por fim, as considerações finais.

\section{VISÃO RELACIONAL DA ESTRATÉGIA}

O conceito de estratégias coletivas, assim como a maior parte dos estudos sobre cooperação interorganizacional na administração, surgiu com maior vigor a partir da década de 1980, fundamentalmente pelos estudos desenvolvidos por Astley (1984) e Astley e Fombrun (1983), Nalebuff e Badenburger (1995) e Child, Faulkner e Tallman (2005) ao mostrarem que as estratégias não precisam se limitar a comportamentos de competição entre atores.

Desde então, as escolas que estudam estratégia têm procurado definir as fontes de vantagens competitivas e de retornos diferenciais de performance. De acordo com Dyer e Singh (1998), durante essa busca, duas perspectivas emergentes têm se tornado proeminente na definição das fontes de vantagens competitivas.

A primeira fonte está associada aos estudos de Porter (1980) sobre a estrutura da indústria, em que os retornos acima da média vem a partir do momento em que a empresa encontrar um posicionamento diferenciado, a partir de uma análise consiste da indústria. A segunda foi idealizada por Barney (1991) e outros autores e foi denominada de Visão Baseada em Recursos (VBR) e tem destacado que as vantagens competitivas de uma empresa advém 


\section{Kadígia Faccin, Ingridi Bortolaso \& Alsones Balestrin}

da posse de recursos distintos. Contudo, apesar de reconhecer que essas duas visões da estratégia tenham contribuído para a compreensão de como as empresas alcançam retornos acima do normal, Dyer e Singh (1998) apontam que elas negligenciaram as relações de cooperação em que a organização está imbricada.

A visão relacional da estratégia tem tido como preocupação empírica a busca do entendimento dos ganhos relacionais percebidos pela atuação conjunta de empresas ou de outros tipos de organizações. A grande mudança apontada pela nova corrente de estudos da estratégia relacional se concentra de forma mais determinante na mudança da unidade de análise, que deixa de ser a competição de mercado e a empresa individual para se concentrar nas relações colaborativas desenvolvidas entre atores (como por exemplo, clientes, concorrentes e fornecedores).

A fim de dar maior ênfase ao uso de estratégias colaborativas Ebers e Jarillo (1998) destacam que, ao adotar a visão relacional, uma organização consegue alcançar e sustentar diferenciais competitivos a partir das seguintes vantagens: a) aprendizado mútuo; b) coespecialidade; c) melhor fluxo de informações; e d) economias de escala. Assim, a visão relacional assume que a unidade de análise seja o relacionamento entre os atores e que a propriedade e o controle das ações sejam atos coletivos, bem como indica que são justamente os investimentos específicos nesse tipo de relacionamento que garantirão um retorno diferenciado à organização.

A mudança de lente teórica provocou uma consequente alteração nos postulados tradicionais estabelecidos no campo de estratégia. Para Zaheer, Gozubuyuk e Milanov (2010) e Ibarra e Hansen (2011), por exemplo, a luta entre entidades autônomas cede lugar às estratégias coletivas e à possibilidade de haver cooperação entre essas entidades para alcançarem seus objetivos estratégicos.

\section{VISÃO RELACIONAL NA IMPLEMENTAÇÃO DE POLÍTICAS PÚBLICAS}

A fim de resolver os complexos problemas de política encarados por Governos, parece ser cada vez mais necessário envolver vários atores nos processos de elaboração e implementação de políticas públicas. Dito de outro modo, evidencia-se ser iminente a necessidade de adoção da visão relacional pelos agentes públicos (KLIJN, 2008; LEYDESDORFF; ETZKOWITZ, 1998). Ou seja, uma estratégia de desenvolvimento econômico ou social deve estar baseada em relações interorganizacionais, as quais, para

REAd | Porto Alegre - Edição 83 - Nº 1 - Janeiro/Abril 2016 - p. 226-251 


\section{A VISÃO RELACIONAL DE POLÍTICAS DE CIÊNCIA E TECNOLOGIA: O CASO DO PROGRAMA CI BRASIL}

Cropper et al. (2008), são relações entre duas ou mais organizações que apresentam objetivos comuns. Logo, parece possível dizer que, para resolver os complexos problemas de políticas públicas encarados pelos Governos, tem se optado cada vez mais pelo estabelecimento de ações colaborativas (ASTLEY; FOMBRUM, 1983).

Para Klijn (2008), a ideia de que a formação e a implementação de políticas públicas ou entrega de serviços aos cidadãos ocorre em uma rede de organizações, baseada em relacionamentos interorganizacionais, certamente não é novidade. Teóricos organizacionais como Emerson (1962), Litwak e Hylton (1962), Saad, Zawdie e Malairaja (2008) e Natário, Couto e Almeida, (2012) ressaltaram as interdependências de organizações na efetivação de resultados positivos no desenvolvimento de territórios.

Para Hans Klijn (2008), a formulação de políticas públicas e implementação das mesmas parece estar repleta de relações interorganizacionais. As evidências empíricas indicam que os Governos de todo o mundo estão enxergando novas formas de governar, sendo a principal delas a interação entre atores, por meio do estabelecimento de relações colaborativas. Importante observar que, quanto mais as políticas públicas forem baseadas em conhecimento técnico e científico, maior a necessidade de formação de arranjos interdisciplinares, que busquem complementar competências distintas para sua eficaz implementação e êxito (BELL, 1968).

Nesse sentido, os Governos podem apoiar a inovação empresarial através do financiamento de pesquisa ou do fornecimento de infraestrutura através de parcerias públicoprivadas (SANDFORT; MILWARD, 2008). A colaboração entre esses atores tem sido apontada como elemento relevante para a produção sistemática de inovações e o desenvolvimento de setores e nações. Freeman (1995) destaca que, na metade do século XIX, existiam pesquisadores que se detinham ao estudo da relação entre o desenvolvimento econômico e a necessidade de articulação entre atores, enfocando principalmente a necessidade de interação entre indústria, ciência e ensino. Isso porque as redes têm sido vistas como uma solução adequada para administrar políticas públicas (FLEURY; OUVERNEY, 2007; DIAS, 2011; LÖFSTRÖM 2010).

Para Lundberg e Andresen (2012), os Governos produzem impacto indireto sobre a forma como as empresas interagem através das normas e regulamentos que afetam a vida empresarial. Por outro lado, Dagnino (2003) reforça a necessidade de interações sociais tais como a cooperação entre Universidade-Governo-Empresa, já que as organizações não são instituições isoladas e os seus objetivos e estratégias emergem sendo constituídos através de múltiplas interações sociais.

REAd | Porto Alegre - Edição 83 - N 1 - Janeiro/Abril 2016 - p. 226-251 
Para Edmondson et al. (2012), o surgimento de uma economia global do conhecimento intensificou a necessidade de parcerias estratégicas que ultrapassem o financiamento tradicional de pesquisa e do desenvolvimento. Ainda segundo os autores, o trabalho conjunto entre universidade e empresa se torna um poderoso motor para a inovação e o crescimento econômico. Alinhados com esse posicionamento, Aiamy e Keshtiaray (2012) argumentam que, por ser capaz de proporcionar resultados, como o desenvolvimento econômico e a geração de renda, a cooperação Universidade-Empresa-Governo tem sido adotada por vários países como base para o desenvolvimento econômico e social.

\section{ESQUEMA TEÓRICO-EMPÍRICO DA PESQUISA}

De forma resumida, a visão relacional proporciona o embasamento teórico para a compreensão do fenômeno analisado, enquanto que o Programa CI Brasil configura-se como o caso de estudo. A Figura 1 ajuda a ilustrar o esquema teórico-empírico que norteia a metodologia e a análise empregadas neste estudo.

O estudo do Programa CI Brasil foi dividido em três categorias de análise: a) antecedentes do Programa CI Brasil; b) dinâmica da colaboração entre Universidade, Indústria e Governo; e, c) principais resultados que já podem ser observados.

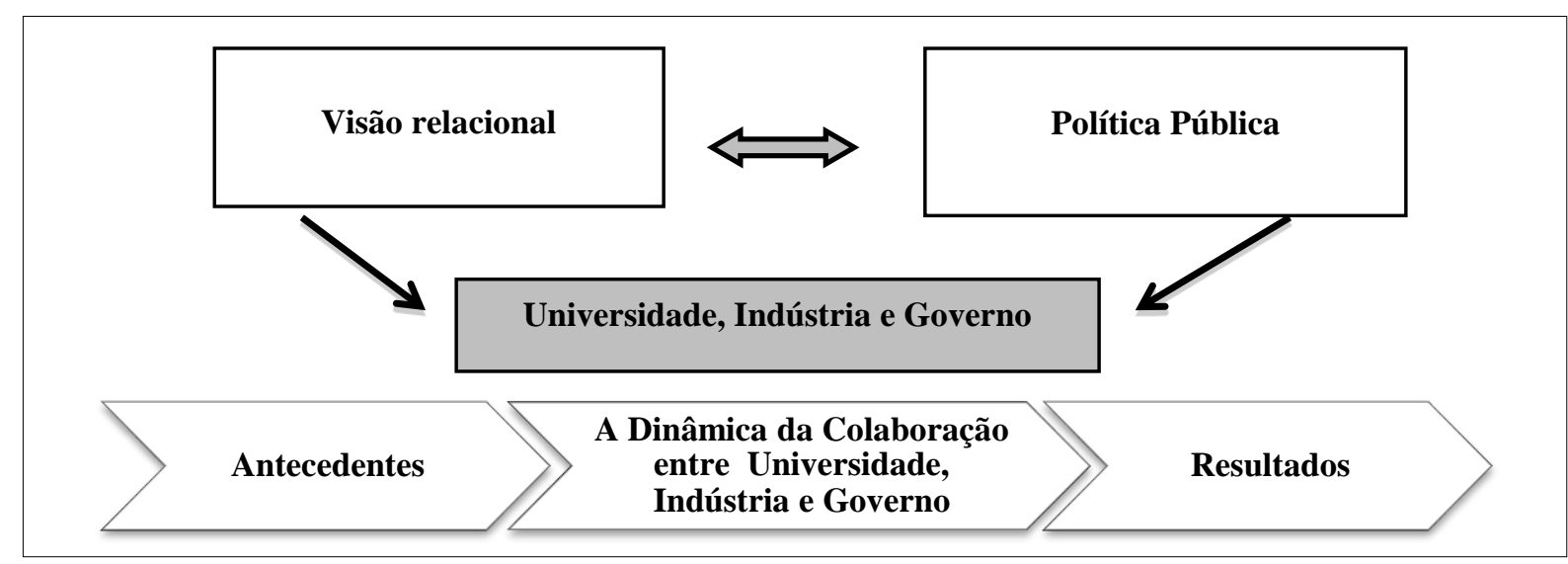

Figura 1- Esquema teórico-empírico da pesquisa

Fonte: Elaborada pelos autores

O primeiro foco de análise do estudo buscou destacar os objetivos do Programa CI Brasil, os principais atores, bem como as características relevantes do mesmo. Já num segundo momento, preocupou-se em investigar o funcionamento do Programa, as suas principais ações e as formas de relacionamento entre os atores responsáveis pela implementação da estratégia governamental. E, de forma a evidenciar como as ações 


\section{A VISÃO RELACIONAL DE POLÍTICAS DE CIÊNCIA E TECNOLOGIA: O CASO DO PROGRAMA CI BRASIL}

colaborativas entre os atores alcançaram êxito, apresentar-se-ão os principais resultados da estratégia colaborativa.

\section{METODOLOGIA}

O Programa CI Brasil, objeto empírico desta pesquisa, foi criado pelo Governo Federal e trata-se de uma política pública, estabelecida na forma de um programa que tem como missão contribuir para a criação e organização de um ecossistema de microeletrônica, visando à inovação em produtos e à inserção do país no mercado de semicondutores. Mais especificamente, o Programa tem como foco formar profissionais capacitados para atender à demanda da indústria de semicondutores, por meio da implementação de centros denominados Design House. Ressalta-se que esses centros são responsáveis pela primeira etapa da complexa cadeia produtiva de semicondutores.

Para a pesquisa de campo foram utilizadas três fontes de dados. Primeiramente, utilizou-se de análise documental a fim de identificar as característica do Programa. Para tanto, foram utilizados documentos como relatórios do Governo e de órgãos vinculados à indústria de semicondutores, bem como os detalhes na concepção do Programa CI Brasil. Além das características e trajetória do Programa, a análise documental proporcionou a identificação de informantes-chave (pesquisadores e especialistas na área de semicondutores).

Com o objetivo de complementar as informações adquiridas, foram conduzidas entrevistas em profundidade com os informantes-chave identificados na etapa de análise documental e com outros pesquisadores vinculados ao Programa CI Brasil, os quais possuem, em média, mais de 15 anos de experiência na indústria de semicondutores e potencial de contribuição para o levantamento de evidências empíricas.

As entrevistas foram conduzidas por meio de roteiro contendo questões semiestruturadas que serviram de guia para nortear as entrevistas de acordo com os objetivos da pesquisa. O roteiro foi pré-testado, com a ajuda de três especialistas vinculados à indústria de semicondutores e ao Programa CI Brasil. O Quadro 1, apresentado a seguir, apresenta alguns dados dos entrevistados. 


\section{Kadígia Faccin, Ingridi Bortolaso \& Alsones Balestrin}

Quadro 1 - Características dos entrevistados

\begin{tabular}{|c|c|c|}
\hline Entrevistado & Organização & Área de Atuação \\
\hline A & Centro de Pesquisa & Pesquisador \\
\hline $\mathrm{B}$ & Programa CI Brasil & $\begin{array}{l}\text { Gerente Nacional do Programa CI- } \\
\text { Brasil }\end{array}$ \\
\hline $\mathrm{C}$ & Secretaria de Política de (SEPIN) & Coordenador Geral de Microeletrônica \\
\hline $\mathrm{D}$ & Design House & Diretor de Tecnologia \\
\hline $\mathrm{E}$ & Design House & Diretor \\
\hline $\mathrm{F}$ & Design House & $\mathrm{CEO}$ \\
\hline $\mathrm{G}$ & $\begin{array}{c}\text { ABINEE - Associação Brasileira da } \\
\text { Indústria Elétrica e Eletrônica }\end{array}$ & Analista \\
\hline $\mathrm{H}$ & Consórcio de Universidades Brasil - IP & Coordenador do Consórcio \\
\hline
\end{tabular}

Fonte: Elaborado pelos autores

Para fins de confidencialidade, os entrevistados serão denominados E1 a E8, de forma aleatória. Na próxima seção, serão discutidos os resultados da pesquisa.

\section{ANÁLISE DOS RESULTADOS}

Apesar de estar entre os cinco maiores mercados mundiais de computadores pessoais, produzindo mais de $70 \%$ do que consome, o Brasil ainda depende da importação de semicondutores e displays para abastecer suas linhas de produção. Os semicondutores representam uma parcela crescente do custo de muitos produtos da indústria de alta tecnologia e o não domínio dessa tecnologia e/ou produção de desses componentes tem um peso muito negativo sobre a indústria brasileira, a balança comercial e a própria soberania nacional.

É pertinente destacar que a indústria de semicondutores é um dos segmentos mais dinâmicos do setor de tecnologias da informação, com múltiplas aplicações nas áreas de entretenimento, educação, segurança industrial, gerando impactos de dimensões culturais e econômicas. As vendas anuais em 2014 atingiram US\$ 335 bilhões anuais de dólares americanos e essa indústria já alcançou recorde de vendas em dois anos consecutivos e está bem posicionada para a continuação do crescimento de 2015 (SIA, 2015).

\subsection{Antecedentes do Programa CI-Brasil}

Destaca-se que essa indústria brasileira de semicondutores já tem sido alvo de fomento no início da década de 60. No entanto, após a queda da reserva de mercado na década de 90 , essa indústria sofreu com a livre concorrência e praticamente foi exterminada no país 


\section{A VISÃO RELACIONAL DE POLÍTICAS DE CIÊNCIA E TECNOLOGIA: O CASO DO PROGRAMA CI BRASIL}

(BORTOLASO et al., 2013). Assim, nos anos 2000 pode-se dizer que essa indústria é novamente alvo de uma segunda onda de incentivos governamentais no sentido de desenvolvê-la. Nessa segunda tentativa, o Banco Nacional de Desenvolvimento Econômico e Social (BNDES), em 2002, contratou um estudo internacional visando à análise e implantação da Indústria de Circuitos Integrados (CI), os populares chips, no país.

De acordo com o entrevistado E1, pesquisador na área de microeletrônica, o estudo contratado pelo BNDES foi de extrema importância para que se compreendesse a complexidade dessa indústria no país. A partir das orientações contidas no estudo de 2002, o Brasil adotou uma série de ações para o desenvolvimento da indústria de semicondutores. Dentre elas, destaca-se o desenvolvimento de um plano para a microeletrônica. Esse plano consistiu em diretrizes básicas para facilitar a implantação dessa indústria. Juntamente com o plano, foram criadas mais duas leis, a lei da Inovação (aprovada em 2004) e a lei do BEM (aprovada em 2005) que também tiveram um papel relevante para o incentivo no desenvolvimento da indústria de alta tecnologia no Brasil.

O relatório final do estudo de 2002 entregue ao BNDES concluiu que a etapa de projeto e concepção, primeira etapa da cadeia produtiva de semicondutores, seria o alvo imprescindível na construção de um ecossistema voltado para a microeletrônica no país (GUTIERREZ; LEAL, 2004). A saber, a cadeia de semicondutores é composta por três etapas: concepção e projeto (design); fabricação (front-end); e c) encapsulamento e teste (back-end).

A etapa de concepção e projeto (design) se ocupa do desenho, ou seja, do projeto do circuito integrado. É nesta fase que é definida a arquitetura do chip. No que compete à etapa intermediária de fabricação (front-end), essa é responsável pela impressão do circuito integrado na lâmina de silício chamada de wafer. A etapa final de produção, encapsulamento e teste (back-end) é responsável pela manufatura do semicondutor e teste das funcionalidades do chip. As etapas de fabricação e encapsulamento demandam altos investimentos em plantas produtivas e são consideradas etapas com alto nível de complexidade. Por outro lado, a etapa de projeto e concepção é destacada como a de menor necessidade de infraestrutura produtiva, sendo concentrada basicamente em recursos humanos altamente qualificados e equipamentos de laboratório.

Segundo o entrevistado E1, uma possível justificativa para a escolha da etapa de projeto pode estar associada ao fato de essa etapa ser uma das mais acessíveis em termos de investimento, “[...] a etapa de front-end é uma parte muito cara e a etapa de back-end também demanda investimentos expressivos". Outro ponto também ressaltado pelo 


\section{Kadígia Faccin, Ingridi Bortolaso \& Alsones Balestrin}

entrevistado reside no fato de o estudo apontar o Governo como o principal ator de fomento. E1 destaca ainda que " [...] a cadeia de semicondutores é uma cadeia muito complexa, muito complicada, depende muito de estratégia e fomento público, de apoio do Governo”.

A ação realizada pelo Governo, voltada à área de concepção e projeto, baseou-se na criação do Programa CI Brasil. Este é vinculado à Secretaria de Política de Informática (SEPIN) do Ministério da Ciência, Tecnologia e Inovação (MCTI). Esse Programa tem como foco formar profissionais capacitados para atender à demanda da indústria de semicondutores. A estrutura do Programa está alicerçada em três eixos: a) incentivar a atividade econômica na área de projetos de Circuitos Integrados (CIs); b) expandir a formação de projetistas de Circuitos Integrados (CIs); e c) promover a criação de uma I indústria nacional de semicondutores (ABDI, 2011).

Na organização das atividades do CI Brasil, participam órgãos do Governo, tais como: Ministério do Desenvolvimento Indústria e Comércio Exterior (MDIC) e Agência Brasileira de Desenvolvimento Industrial (ABDI). Participam também as agências de fomento Financiadora de Estudos e Projetos (FINEP), Conselho Nacional de Desenvolvimento Científico e Tecnológico (CNPq), Banco Nacional de Desenvolvimento Econômico e Social (BNDES) e sociedades científicas, tais como: a Sociedade Brasileira de Microeletrônica (SBMicro) e a Sociedade Brasileira de Computação (SBC). As sociedades empresariais são representadas pelas entidades Abinee - Associação Brasileira da Indústria Elétrica e Eletrônica (ABINEE) e Centro de Excelência em Tecnologia Eletrônica Avançada (CEITEC S.A.). Ainda vinculada ao Programa CI Brasil, apoiam também o consórcio Brazil-IP, que conta com a participação de 18 universidades nacionais. Para o entrevistado E2, “[...] o CI é uma ação conjunta entre o Governo Federal, empresas e o setor acadêmico, com o objetivo desenvolver um ecossistema em microeletrônica”.

Percebe-se pelo número de organizações envolvidas no desenvolvimento do Programa CI Brasil a importância da cultura setorial ou sistemas de significado compartilhados que, segundo Klijn (2008), são importantes para o sucesso das interações já que os atores dentro de políticas setoriais compartilham percepções da importância do setor ou de normas ou crenças profissionais.

Quanto a implantação do Programa CI Brasil, tornou-se possível devido a uma ação conjunta e articulada entre Universidade, Indústria e Governo Federal, o que pode ser entendida como uma ação colaborativa (ASTLEY; FOMBRUM, 1983). Assim, parece possível afirmar que o Governo Brasileiro segue em direção a arranjos colaborativos para atingir resultados mais expressivos no desenvolvimento da indústria de semicondutores. A 


\section{A VISÃO RELACIONAL DE POLÍTICAS DE CIÊNCIA E TECNOLOGIA: O CASO DO PROGRAMA CI BRASIL}

ação nacional mostra-se similar à destacada por Klijn (2008) em seu estudo. Este autor enfatizou que as redes funcionam como veículos para atingir os produtos de políticas e organizar a entrega de serviços, conectando diversos atores.

Ainda segundo o mesmo entrevistado (E2) a necessidade da intervenção do Governo como articulador dessa cadeia não é uma prerrogativa somente brasileira: “[...] não é só aqui... é em todo o lugar no mundo, o Governo geralmente atua como fomentador, então o Brasil está caminhando para isso, está fazendo ações disso”. De acordo com artigos recentes, muitos países têm utilizado estratégias colaborativas para alavancar o desenvolvimento da indústria de semicondutores. O Quadro 2 apresenta os principais elementos antecedentes da criação do Programa CI Brasil.

Quadro 2 - Evidências Empíricas

\begin{tabular}{|c|c|}
\hline & $\begin{array}{l}\text { "O estudo contratado pelo BNDES foi de extrema importância para se entender a } \\
\text { complexidade dessa indústria no país. (EI). }\end{array}$ \\
\hline \multirow{4}{*}{$\begin{array}{l}\text { Evidências } \\
\text { Empíricas sobre os } \\
\text { antecedentes do } \\
\text { Programa CI Brasil }\end{array}$} & $\begin{array}{l}\text { "A etapa de front-end é uma parte muito cara e a etapa de back-end também } \\
\text { demanda investimentos expressivos" (E1). }\end{array}$ \\
\hline & $\begin{array}{l}\text { "Cadeia de semicondutores é uma cadeia muito complexa, muito complicada, } \\
\text { depende muito de estratégia e fomento público, de apoio do Governo" (E2). }\end{array}$ \\
\hline & $\begin{array}{l}\text { "Não é só aqui... é em todo o lugar no mundo, o Governo geralmente atua como } \\
\text { fomentador, então o Brasil está caminhando para isso, está fazendo ações } \\
\text { disso"(E3). }\end{array}$ \\
\hline & $\begin{array}{l}\text { "E mais, como há um elevado risco na atividade, o apoio do Governo é } \\
\text { fundamental"(E6). }\end{array}$ \\
\hline
\end{tabular}

Fonte: Elaborado pelos autores

A partir dos antecedentes do Programa CI Brasil, pode-se evidenciar que vários fatores contribuíram para a criação de um ecossistema para o desenvolvimento da indústria de semicondutores. Dentre esses fatores, é possível destacar o estabelecimento de estratégias de cooperação entre diferentes instituições para o estabelecimento de um programa voltado a primeira etapa da cadeia de semicondutores.

\subsection{Dinâmica da Colaboração}

O Programa CI Brasil opera via ação conjunta entre Universidade, Indústria e Governo Federal com vistas a desenvolver a indústria de microeletrônica no país. Com esse intuito, são disponibilizados pelo Programa CI Brasil: a) bolsas de estudo-pesquisa por meio do Conselho Nacional de Desenvolvimento Científico e Tecnológico (CNPQ), que variam de R $\$ 2.000,00$ a R \$ 8.000,00; b) investimento em infraestrutura, como servidores e software, através da 


\section{Kadígia Faccin, Ingridi Bortolaso \& Alsones Balestrin}

disponibilização de licenças; e c) treinamento nas ferramentas de projeto viabilizado via Financiadora de Estudos e Projetos (FINEP), sendo que os produtos gerados pelas Design Houses podem ser financiados pela FINEP e pelo Banco Nacional de Desenvolvimento Econômico e Social (BNDES).

O Programa funciona por meio de editais e chamadas públicas. Assim, uma das suas primeiras ações foi a seleção pública de propostas para a ampliação do número de centros de projetos de Circuitos Integrados (Design Houses - DHs), de acordo com o edital MCT/CNPQ/CI Brasil no 59/2008. As propostas aprovadas foram financiadas com valores oriundos de fundos setoriais, liberados conforme a disponibilidade orçamentária durante os anos de 2008 e 2009. Até o momento, a maior parte das ações tem dependido de financiamento do Governo Federal, sendo que já foram realizados investimentos superiores a 100 milhões de reais, desde 2005, na estruturação do sistema de microeletrônica.

Com o apoio do Governo Federal, operam no Brasil 22 Design Houses, as quais são participantes do Projeto CI Brasil (MCTI, 2011). Destas, sete nasceram na primeira fase do Programa. Segundo o entrevistado E1, “[...] treze são apoiadas parcialmente pelo Programa e não possuem fins lucrativos, 5 recebem apoio do Governo, mas possuem fins lucrativos e 4 são Design Houses independentes”. As informações fornecidas pelo entrevistado foram confirmadas pelos relatórios oficiais do Programa. Em sua maioria, são spin-offs, que surgiram ou estão conectadas a universidades ou a instituições públicas de pesquisa.

Quando o foco de análise se volta para as esferas vinculadas ao Programa CI Brasil, pode-se utilizar o depoimento de um entrevistado para compreender as relações entre as três esferas. Ao ser questionado a respeito do papel da universidade, o entrevistado E2 informa: "[...] o papel da universidade é formar recursos humanos e realizar atividades de pesquisa e desenvolvimento, somando-se às atividades das instituições e centros de $P \& D ”$. Esta evidência está de acordo com o trabalho de Leydesdorf e Etzkowitz (2000), o qual indica uma nova função da universidade: produzir conhecimento associado aos problemas do setor empresarial.

No que tange ao papel da indústria, o mesmo entrevistado, E2, gerente nacional do Programa CI Brasil, explica que “[...] a indústria tem o papel de destino dos profissionais de alto nível formados e, além da importância econômica, há ainda a demanda cada vez maior por desenvolver e incorporar nos produtos tecnologia e inovação, como forma de ampliar a produtividade e competitividade. E a inovação é introduzida nos produtos pelos componentes e pelo software". E, quando detalhado o papel do Governo, novamente é ressaltada a 


\section{A VISÃO RELACIONAL DE POLÍTICAS DE CIÊNCIA E TECNOLOGIA: O CASO DO PROGRAMA CI BRASIL}

responsabilidade como fomentador, algo já destacado anteriormente pelo entrevistado E1 e pelo estudo realizado em 2002 pelo BNDES. Para o entrevistado E3, coordenador geral de microeletrônica, "O Governo está preocupado com o fortalecimento do setor produtivo, formação de recursos humanos e incremento das atividades de P\&D. Claro que o déficit da balança comercial preocupa, mas a evolução e o impacto das TIC na economia moderna ainda está longe de ser avaliado. E mais, como há um elevado risco na atividade, o apoio do Governo é fundamental”. A Figura 2 busca sistematizar a dinâmica do Programa CI Brasil.

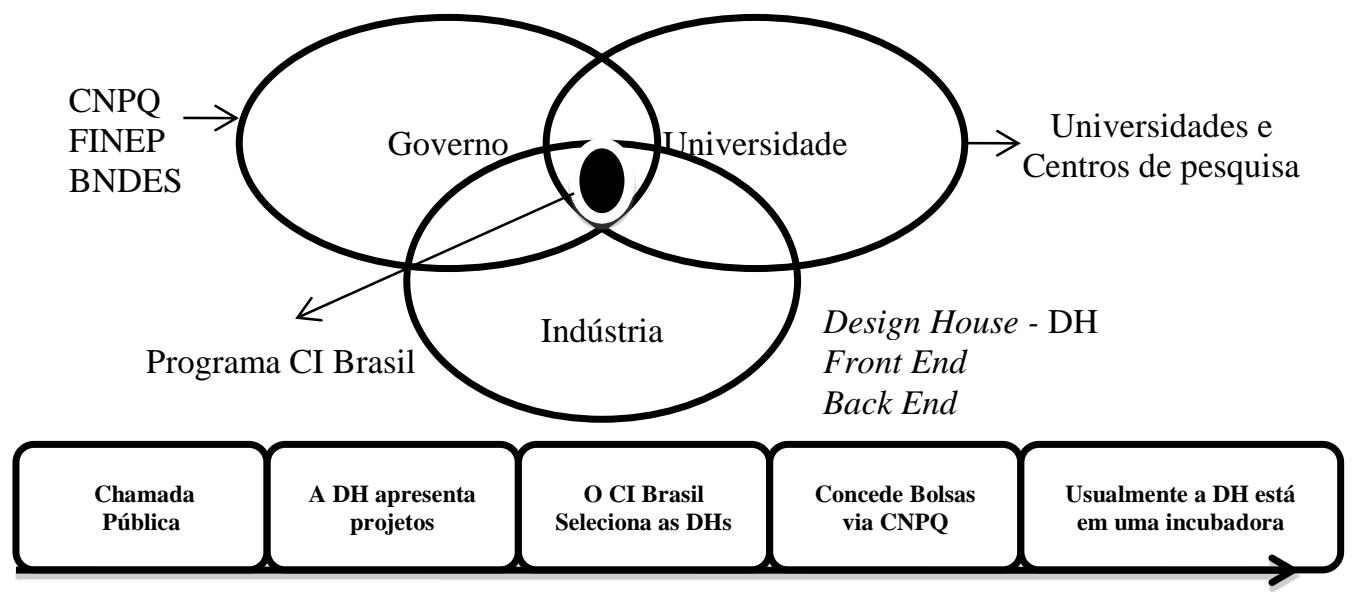

Figura 2- Dinâmica da Colaboração do Programa CI Brasil

Fonte: Elaborado pelos autores

Para o Programa CI Brasil, o Governo vem utilizando uma estratégia baseada em relações interorganizacionais, especialmente pela interdependências entre os atores envolvidos no processo. Portanto, eles devem interagir com outras organizações, a fim de complementar recursos. Estes podem ser financeiros, tecnológicos, legitimação de autoridade ou conhecimento. Conforme já foi destacado por Klijn (2008) e Rhodes (1988) tais redes se desenvolvem porque essas a complementaridade de recursos continuam através do tempo.

Juntamente com o incentivo ao nascimento e à manutenção das Design Houses, o Programa também opera no desenvolvimento de recursos humanos para serem absorvidos pelas DHs. Isso porque a principal exigência de uma empresa de circuitos integrados prendese à disponibilidade de profissionais qualificada (ABDI, 2011; MCTI, 2011). Assim, no que se refere à formação de mão de obra qualificada, foi criado, em 2008, o Programa Nacional de Formação de Projetistas de Circuitos Integrados (PNF-PCI). O PNF-PCI foi constituído em parceria com a Cadence Design Systems, uma das líderes mundiais no segmento, tendo como objetivo oferecer ao país profissionais especializados em projetar circuitos integrados. Cabe observar que, ao PNF-PCI, estão vinculados dois centros de treinamento.

REAd | Porto Alegre - Edição 83 - N 1 - Janeiro/Abril 2016 - p. 226-251 
O objetivo dos centros de treinamento de projetistas em CIs é o estabelecimento de um ciclo contínuo de capacitação, implantação, desenvolvimento e consolidação da área de microeletrônica no país. Essa medida visa ofertar recursos humanos qualificados e em condições equivalentes às oferecidas por países como Índia, China, Rússia e países do leste europeu. Podem participar dos processos seletivos profissionais egressos de curso superior em áreas como Engenharia Elétrica, Eletrônica, Telecomunicações, Computação, Física e áreas afins, tendo como benefícios uma bolsa mensal de R \$2.000,00 do CNPQ e a participação no Programa de Trainees nas DHs vinculadas ao CI Brasil. Atualmente, o Programa mantém dois centros de treinamento nacionais, um deles em Porto Alegre (RS) e o outro em Campinas (SP). O primeiro centro de treinamento foi estruturado em março de 2008 e utilizou como base a estrutura física do instituto de informática da Universidade Federal do Rio Grande do Sul (UFRGS). A operação e a montagem da infraestrutura do centro foram viabilizados por meio de um financiamento oriundo da Financiadora de Estudos e Projetos (FINEP). A cidade de Campinas abriga o segundo centro de treinamento, que está localizado no centro de Tecnologia da Informação Renato Archer.

Ainda, em novembro de 2007, foi instituído o SIBRATEC (Sistema Brasileiro de Tecnologia), uma rede de centros de inovação em microeletrônica operada pela Financiadora de Estudos e Projetos (FINEP). Entre suas atribuições está o encaminhamento de projetos cooperativos que serão desenvolvidos para atender demandas específicas de empresas. Os primeiros projetos conjuntos apoiados pela rede se referem à seleção realizada em maio de 2011, com uma demanda total de recursos na ordem de $\mathrm{R} \$ 41,5$ milhões.

Além das ações já destacadas, a estratégia conta ainda com o apoio do consórcio Brazil-IP. O Brazil-IP (Brazil Intelectual Property) é um consórcio de laboratórios e universidades brasileiras que visa à formação de recursos humanos em projeto de circuitos integrados. O objetivo central do consórcio Brazil-IP é a formação de recursos humanos no projeto de circuitos integrados de qualidade através do estabelecimento de um programa de treinamento que inclui uso de ferramentas comerciais, cursos de capacitação e projetos de circuitos usados largamente na indústria. Essas atividades têm como foco alunos de graduação.

Foi criado ainda o "Programa de Capacitação das DHs Brasileiras", que visa capacitar as Design Houses em gestão empresarial. Segundo a analista responsável pelo acompanhamento dos eventos ligados ao Programa CI Brasil, são desenvolvidas ações de sensibilização, citando como exemplo um Workshop realizado em setembro de 2012, que teve uma oficina de sensibilização sobre o caso das DHs portuguesas. Além disso, foram 


\section{A VISÃO RELACIONAL DE POLÍTICAS DE CIÊNCIA E TECNOLOGIA: O CASO DO PROGRAMA CI BRASIL}

selecionados gestores de 10 DHs para realizarem o EMPRETEC, que consiste em uma metodologia desenvolvida pela Organização das Nações Unidas (ONU) para desenvolver o empreendedorismo.

A Agência Brasileira de Desenvolvimento Industrial (ABDI) e a Associação Brasileira de Private Equity e Venture Capital (ABVCAP) firmaram convênio de cooperação, com o apoio da FINEP, detentora de larga experiência em Venture Capital, e promoveram um Venture Fórum específico para as empresas vinculadas ao setor de Microeletrônica e também Tecnologia da Informação. Outra ação que tem características de marco regulatório da mudança organizada pelo Governo Federal é o PADIS (Programa de Apoio ao Desenvolvimento Tecnológico da Indústria de Semicondutores e Displays). Por ser uma importante indutora do desenvolvimento tecnológico, a indústria de semicondutores recebe atenção especial do Governo Brasileiro por meio do Plano Brasil Maior, que é a política industrial, tecnológica e de comércio exterior do governo federal. Para aumentar a competitividade no segmento e atrair indústrias estrangeiras de componentes eletrônicos, o Governo criou o Programa de Apoio ao Desenvolvimento da Indústria de Semicondutores e Displays (PADIS), que reúne incentivos para desonerar a produção e a exportação.

O PADIS oferece incentivo às empresas que, como contrapartida, têm que investir pelo menos $5 \%$ do faturamento local em pesquisa e desenvolvimento. Outro mecanismo de apoio oferecido pelo Governo é a disponibilização de linhas de financiamento do BNDES para viabilizar projetos das Design Houses e as demais etapas da cadeia de produção, como Front-End (wafer fab) e Back-End (assembling \& testing). Dessa maneira, objetivando a implantação, institucionalização e operacionalização do PADIS, o Governo criou um plano de ações com as principais estratégias e metas que deveriam ser atingidas entre 2007 e 2010: a PACTI - Política de Ação de Ciência e Tecnologia para o Desenvolvimento Nacional. O

Quadro 3 apresenta outras evidências empíricas sobre a dinâmica de colaboração para a implementação do Programa. 


\section{Kadígia Faccin, Ingridi Bortolaso \& Alsones Balestrin}

Quadro 3 - Dinâmica de colaboração entre os atores

\begin{tabular}{|c|c|}
\hline & "A estratégia vinculando esses três 'agentes'é fundamental"(E1). \\
\hline \multirow{3}{*}{$\begin{array}{l}\text { Evidências Empíricas } \\
\text { sobre a dinâmica de } \\
\text { colaboração entre os } \\
\text { atores institucionais no } \\
\text { Programa CI Brasil }\end{array}$} & $\begin{array}{l}\text { "O papel da universidade é formar recursos humanos e realizar atividades de } \\
\text { Pesquisa e Desenvolvimento, somando-se às atividades das instituições e centros de } \\
P \& D "(E 2) \text {. }\end{array}$ \\
\hline & $\begin{array}{l}\text { "A indústria tem o papel de destino dos profissionais de alto nível formados e, além } \\
\text { da importância econômica, há ainda a demanda cada vez maior por desenvolver e } \\
\text { incorporar nos produtos de tecnologia e inovação, como forma de ampliar a } \\
\text { produtividade e a competitividade. E a inovação é introduzida nos produtos pelos } \\
\text { componentes e software"(E4). }\end{array}$ \\
\hline & $\begin{array}{l}\text { "O Governo está preocupado com o fortalecimento do setor produtivo, formação de } \\
\text { recursos humanos e incremento das atividades de } P \& D "(E 2) \text {. }\end{array}$ \\
\hline
\end{tabular}

Fonte: Elaborado pelos autores

Na próxima seção, serão apresentados os principais resultados do Programa CI Brasil.

\subsection{Resultados do Programa CI Brasil}

Desde sua implantação no ano de 2005, o Programa CI Brasil apresenta resultados expressivos, como o desenvolvimento de projetos em geral de forma cooperativa entre as Design Houses, formação de mão de obra qualificada e disponibilização de treinamento no que tange à gestão das DHs. É importante ressaltar que esses resultados estão vinculados a oito anos de Programa e a apenas duas chamadas públicas para seleção e apoio à Design House. Também cabe destacar que, apesar dos vários profissionais qualificados pelos centros de treinamento, eles só iniciaram os treinamentos em 2008.

A ação mais importante do programa estratégico para o setor de semicondutores existente na PITCE (Política industrial, Tecnológica e de Comércio Exterior) foi a criação da CEITEC S.A., o Centro de Excelência em Tecnologia Eletrônica Avançada, uma empresa pública federal situada em Porto Alegre no Rio Grande do Sul. O investimento de mais de meio bilhão de reais feito na CEITEC S.A. objetiva o desenvolvimento da indústria eletrônica brasileira através da implantação de uma base sólida no setor de semicondutores (CEITEC, 2012).

A respeito dos projetos de forma geral, pode-se citar o "Chip do Boi”, desenvolvido pela CEITEC S.A., que é um componente básico dos brincos eletrônicos usados na identificação e rastreabilidade do gado bovino. Outro projeto que merece destaque é o projeto da Floripa DH (Design House) que vem desenvolvendo um circuito integrado para dispositivos de auxílio a deficientes auditivos em parceria com as empresas LSI-TEC, de São Paulo, e Amplivox, de Pelotas/RS (REVISTA AMANHÃ, 2012). Além disso, a SMDH 


\section{A VISÃO RELACIONAL DE POLÍTICAS DE CIÊNCIA E TECNOLOGIA: O CASO DO PROGRAMA CI BRASIL}

(Design House) desenvolveu, em parceria com a Chipus (Design House), um microcontrolador utilizado em equipamentos de segurança, batizado de ZR16. Este é considerado o primeiro chip microcontrolador com projeto $100 \%$ brasileiro (FACCIN, BALESTRIN e BORTOLASO, 2016). Segundo o entrevistado E4, “[...] esses projetos são financiados a fundo perdido". Além destes, pode-se destacar também os primeiros projetos cooperativos apoiados pela rede SIBRATEC, que estão disponibilizados no Quadro 4 e referem-se à seleção realizada em maio de 2011.

Quadro 4 - Empresas Contempladas com Financiamento FINEP e Parceiras

\begin{tabular}{|c|c|c|}
\hline Projeto & Empresa Proponente & Parceiro(s) \\
\hline Plataforma de Ips & CHIPUS & UFSM \\
\hline R FID Tag passivo para medicina & INOVACODE & UFRGS \\
\hline $\begin{array}{c}\text { CODEC-PQ - compressão e descompressão de sinais } \\
\text { e informações de qualidade da energia elétrica. }\end{array}$ & Embrasul & LSTI-Tec \\
\hline $\begin{array}{c}\text { Sensor EE: sinalizador de falhas em sistema de } \\
\text { distribuição elétrica }\end{array}$ & Expertec \\
\hline $\begin{array}{c}\text { EM-Hard "energyharvesting" carregador solar de } \\
\text { bateria. }\end{array}$ & SiliconReef & Cesar e CTI Renato Archer \\
\hline IDTV: chip de recepção para TVD & Idea & Eldorado e CTI Renato Archer \\
\hline Sistema de Semáforos Verdes & SeriTel & Cetene \\
\hline
\end{tabular}

Fonte: MCTI (2011)

No que se refere à formação de recursos humanos, o Programa CI Brasil, por meio dos centros de treinamento, já formou, de 2008 até 2011, mais de 500 projetistas de circuitos integrados entre sistemas digitais, Mixed-Signal e Radiofrequência. Em média, formam-se 140 projetistas a cada ano no país. Cerca $50 \%$ dos egressos anuais dos centros de treinamento são absorvidos pelas DHs vinculadas ao Programa CI Brasil.

Tais evidências vão ao encontro do trabalho de Geddes (2008), ao indique que ao se estabelecer uma parceria, reúnem-se todas as chaves e interesses das organizações envolvidas no desenvolvimento urbano e regional. De muitas formas, a competência da parceria é multidimensional, na qual um dos principais propósitos dos arranjos é unir setorialmente atividades específicas que não sejam tão bem sucedidas quando realizadas isoladamente cada uma por si. No que tange ao financiamento das ações colaborativas, as parcerias podem apresentar uma base de recursos diferenciados, não só em termos de quantidade de recursos disponíveis, como também de suas fontes e sua natureza.

Outro resultado do Programa centra-se na qualificação da gestão das Design Houses. O CI Brasil fornece acesso ao "Programa de Capacitação das DHs Brasileiras", que visa capacitar as Design Houses em gestão empresarial. Conforme a analista responsável pelo 


\section{Kadígia Faccin, Ingridi Bortolaso \& Alsones Balestrin}

acompanhamento dos eventos ligados ao Programa CI Brasil, são desenvolvidas ações de sensibilização, citando como exemplo o Workshop realizado em setembro de 2012, que teve uma oficina de sensibilização sobre o caso da DHs portuguesas.

No que tange aos resultados vinculados ao Programa Brazil-IP, tem-se a formação de engenheiros graduados com conhecimento em metodologias de projetos, ferramentas de EDA comerciais, e que tenham sido confrontados com projetos reais prototipados em tecnologia FPGA(Field-programmable gate array) e Silício. De acordo com dados fornecidos pela coordenação do Projeto Brazil-IP, no período de julho de 2008 a novembro de 2012, foram projetados cerca de 18 IP-cores segundo padrões industriais de qualidade. Além disso, foi formado neste período um grupo de aproximadamente 120 alunos capacitados para o Projeto de IP-cores de qualidade industrial. As informações da coordenação disponibilizadas às pesquisadoras ainda destacam que esse grupo apresenta um alto fator multiplicativo: "Se considerarmos o fator multiplicativo deste grupo nas instituições, podemos ter mais de 120 profissionais treinados" (dados secundários da pesquisa). Esse fator multiplicativo ao qual a coordenação do Programa se refere é um dos benefícios da pesquisa que está se espalhando pela comunidade como um todo de forma dinâmica. Autores como Todling, Lehner e Kaufmann (2009) encontraram resultados semelhantes em seus estudos.

Apenas 5\% do total de egressos tiveram suas bolsas canceladas por mau desempenho durante o curso de graduação. E, dos concluintes, apenas $8 \%$ não estão atuando na área de microeletrônica. Contudo, a grande maioria dos egressos (90\% do total) concluiu seus cursos e está atuando na área de microeletrônica. Uma grande parte dos egressos está atuando em uma das atividades do CI Brasil (28\% em alguma DH do CI Brasil e 23\% em um dos Centros de Treinamento do CI Brasil). Pode-se concluir que, com relação à atração de alunos para a área de microeletrônica, o Brazil-IP tem tido um papel de destaque, uma vez que $90 \%$ dos egressos continuaram atuando na área de microeletrônica após a participação no Programa Brazil-IP e tiveram uma rápida absorção pelo mercado de trabalho nacional. Pode-se ainda complementar dizendo que as universidades do consórcio apresentam a função empreendedora.

Cabe explicar que a universidade empreendedora é aquela que incorpora na sua missão o desenvolvimento do empreendedorismo (LEYDESDORFF; ETZKOWITZ, 1995; 1997; 2000). Nesse sentido, as universidades passam a atuar fortemente em conjunto com as empresas e o Governo, transferindo tecnologia efetiva por meio da capitalização do conhecimento para a sociedade (LEYDESDORFF; ETZKOWITZ, 1995). Percebe-se, então, que a capitalização do conhecimento que vai gerar inovações no setor de semicondutores foi 


\section{A VISÃO RELACIONAL DE POLÍTICAS DE CIÊNCIA E TECNOLOGIA: O CASO DO PROGRAMA CI BRASIL}

entendida pelos responsáveis pelas ações do Programa CI Brasil como um processo que envolve mecanismos de feedback e relações interativas entre a ciência, a tecnologia, o aprendizado, a produção e a política.

Quadro 5 - Evidências empíricas sobre os resultados do Programa CI Brasil

\begin{tabular}{|c|c|}
\hline & $\begin{array}{l}\text { "A [...] corporação nacional, é o fabricante de semicondutores, nós aqui no Brasil } \\
\text { somos basicamente um braço de projeto mundial, é uma das design centers que temos } \\
\text { no mundo[...] nossodesign é competitivo mundialmente" (E7). }\end{array}$ \\
\hline & $\begin{array}{l}\text { "[...] a gente tem projeto hoje sendo desenvolvido em parceria com a França e a } \\
\text { Alemanha. É claro que a gente tem condiçôes de concorrer mundialmente e já } \\
\text { estamos fazendo isso"(E7). }\end{array}$ \\
\hline & $\begin{array}{l}\text { "É bom o CI, porque o Programa, a ação do Programa é excelente. Ainda pode } \\
\text { melhorar, mas para começar tem dado bons resultados"(E8). }\end{array}$ \\
\hline & $\begin{array}{l}\text { "[...] o Brasil entrou na rota desses dois empresários em função do Programa de } \\
\text { Apoio à Iindústria de Semicondutores, que é o PADIS. Então esse Programa foi o } \\
\text { forte indutor para que esses dois empresários decidissem pelo Brasil" (E2). }\end{array}$ \\
\hline $\begin{array}{l}\text { Evidências } \\
\text { Empíricas sobre os } \\
\text { resultados do } \\
\text { Programa CI Brasil }\end{array}$ & $\begin{array}{l}\text { "A CEITEC é uma realidade que vem se concretizando [...] como pioneira na parte } \\
\text { de front-end e na parte de projetos de CI, ela tem a missão de desenvolver, capacitar } \\
\text { recursos humanos, desenvolver mercado, atrair elos da cadeia, desenvolver } \\
\text { fornecedores e prestadores de serviços nacionais, ela tem que capacitar o meio, que } \\
\text { se traduz na missão fundamental do Governo, que é fundamental à criação e à } \\
\text { consolidação do ecossistema no Brasil" (E6). }\end{array}$ \\
\hline & $\begin{array}{l}\text { "Do ponto de vista de oferta de produto, comparável à concorrência internacional, a } \\
\text { CEITEC já desenvolveu e disponibilizou no mercado um produto que poucos tem no } \\
\text { mundo, e se torna competitivo em nível global"(E6). }\end{array}$ \\
\hline & $\begin{array}{l}\text { "Todos são bolsistas, ou quase todos, se não fosse dessa forma, nós não teríamos } \\
\text { como dar vida ao projeto. A concessão da bolsa é fundamental"(E1). }\end{array}$ \\
\hline & $\begin{array}{l}\text { "O centro de treinamento funciona como uma forma de padronizar os conhecimentos } \\
\text { que são úteis no desenvolvimento desta indústria"(E2). }\end{array}$ \\
\hline
\end{tabular}

Fonte: Elaborado pelos autores

A respeito da internacionalização da indústria brasileira de semicondutores, é possível citar a importante contribuição dos investimentos externos diretos. Esse tipo de investimento garantiu a instalação da maior fábrica de encapsulamento de semicondutores da América Latina em território Brasileiro. A HT Micron, join-venture formada pela Hana Micron, da Coreia do Sul, e a holding brasileira Parit Participações está situada em São Leopoldo, dentro do Parque Tecnológico da Universidade do Vale do Rio dos Sinos (Tecnosinos). Os investimentos previstos são de $\mathrm{R} \$ 200$ milhões, sendo que estes recursos têm apoio do BNDES e da FINEP.

Dessa forma, a partir da descrição das ações e dos principais resultados do Programa CI Brasil, parece possível concluir que o Governo Nacional está utilizando uma estratégia coletiva para fomento da polícia pública e desenvolvimento do setor de semicondutores nacional. A Figura 3 apresenta a síntese dos antecedentes, dinâmica de cooperação e resultados do Programa CI Brasil. 


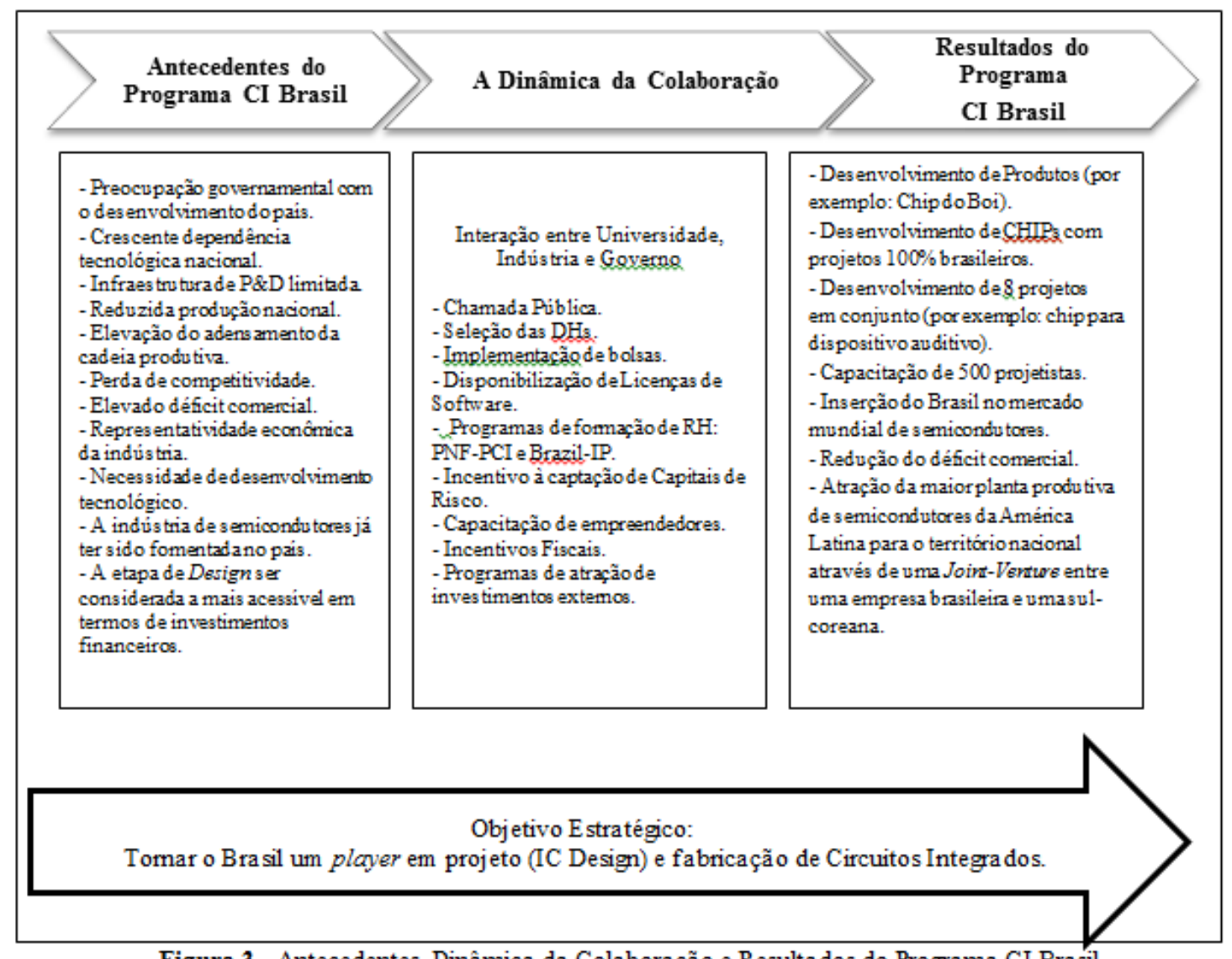

Figura 3 - Antecedentes, Dinâmica da Colaboração e Resultados do Programa CI Brasil

Fonte: Elaborado pelos autores

Percebe-se uma grande participação, tanto na questão financeira, como na gestão por parte do Governo, além de uma ampla interação entre universidade e indústria. Etzkowitz (2009) destacou que raramente a força empregada pelos atores é igual: uma geralmente serve como força motivadora, a organizadora da inovação, em torno da qual as outras contribuem, como é o caso do Governo no Programa CI Brasil. Embora mantenha ações colaborativas com a universidade e a indústria, é ele quem serve como força motriz, estando na posição de "gestor de rede". Embora esteja nessa posição, vale observar que as suas decisões não são unilaterais visto que elas são tomadas de forma conjunta entre os atores que se demonstram altamente interdependentes no processo de desenvolvimento da indústria de semicondutores. 


\section{A VISÃO RELACIONAL DE POLÍTICAS DE CIÊNCIA E TECNOLOGIA: O CASO DO PROGRAMA CI BRASIL}

\section{CONSIDERAÇÕES FINAIS}

A partir do caso estudado, pode-se observar que a elaboração e implementação da política pública em análise envolveu uma gama de relações entre Universidade, Indústria e Governo, buscando o desenvolvimento da indústria nacional de semicondutores. Isso, de certa forma, contrapõe os preceitos clássicos da gestão pública, que fortemente enfatiza a tomada de decisões e implementação de políticas públicas a partir de uma visão unilateral e sem maior interação com os atores envolvidos. As conversações teóricas e as evidências empíricas aqui apresentadas sugerem que a implementação de políticas públicas, no caso específico do Programa CI Brasil, foi embasada fortemente na adoção de estratégias relacionais.

A visão relacional da estratégia na implementação de políticas públicas, por sua vez, foca na relação entre os que tomam decisões políticas e como os demais atores são organizados na operacionalização da estratégia. Em suma, o papel do Governo, a partir de uma perspectiva relacional estabelecida foi equivalente ao de um articulador e facilitador da construção da política pública.

Parece possível dizer que o Programa CI Brasil, organizado com a finalidade de garantir que Universidade, Indústria e Governo estivessem engajados num mesmo objetivo, está cumprindo com algumas metas principais, dentre as quais pode-se evidenciar o avanço da indústria de semicondutores a partir das suas inter-relações, que destacam um modelo não linear de implementação de política pública de desenvolvimento de um determinado setor econômico.

A estratégia de ações colaborativas, identificada na descrição do Programa e dos seus resultados, está apresentando importantes avanços, uma vez que incentiva a atividade econômica na área de projetos de circuitos integrados. Outros sim, espera-se nos próximos anos que ocorra um aumento do Produto Interno Bruto (PIB) gerado pela Indústria da Microeletrônica, que hoje não passa de $2 \%$ no Brasil. Além disso, tem se expandido a formação de projetistas de CIs e, em consequência, vem se buscando o adensamento do complexo eletrônico nacional; atraindo, inclusive, investidores e empresas multinacionais.

A partir da realização deste estudo, tornou-se possível descrever os antecedentes e os resultados alcançados pelo Programa CI Brasil, bem como a dinâmica relacional de colaboração entre os seus três atores institucionais - U-I-G. Dentre os principais antecedentes, foi possível evidenciar a preocupação governamental com o desenvolvimento do país e a crescente dependência tecnológica, somada à perda de competitividade e ao elevado déficit comercial do setor de semicondutores.

REAd | Porto Alegre - Edição 83 - N 1 - Janeiro/Abril 2016 - p. 226-251 
Para concluir este estudo enfatizou que o desenvolvimento da indústria de semicondutores dá mostras de estar especialmente dependente da cooperação e interação e que os primeiros resultados já podem ser observados e são considerados satisfatórios. Existem indícios de relações colaborativas e não tradicionais na condução da política pública de desenvolvimento do setor de semicondutores. Por conseguinte, dado que esta pesquisa teve caráter apenas exploratório para o fenômeno, a estratégia baseada em ações colaborativas para desenvolver um setor baseado na dinâmica do conhecimento e da inovação deve ser foco de estudos futuros mais aprofundados.

\section{REFERÊNCIAS}

ABDI. Agência Brasileira de Desenvolvimento Industrial. As Design Houses (DHs) Brasileiras. Relatório Analítico. Brasília, Novembro, 2011.

AIAMY, M.; KESHTIARAY, N. A perspective of the cooperation between university and industry at Islamic Azad University, Sanandaj Branch, and its comparison with Kingston University London. Procedia - Social and Behavioral Sciences, v. 46, p. 2509-2513, Janeiro, 2012.

ASTLEY, W.G. Toward an appreciation of collective strategy. Academy of Management Review, [s.1.], v. 9, n. 3, p. 526-535, 1984.

ASTLEY, W.G; FOMBRUN, C. J. Collective Strategy: Social Ecology of Organizational Environments. The Academy of Management Review, v. 8, n. 4, p.576, 1983.

BARNEY, J. Firm resources and sustained competitive advantage. Journal of Management, Greenwich, v. 17, n. 1, p. 99-120, 1991.

BARNEY, J. B.; KETCHEN, D. J.; WRIGHT, M. The Future of Resource-Based Theory: Revitalization or Decline? Journal of Management, v. 37, n. 5, p. 1299-1315, 10 mar. 2011.

BELL, D. The measurement of knowledge and technology. In: SHELDON, E.B., MOORE W.E. (Eds). Indicators of Social Change: Concepts and Measurements. Russel Sage Foundations, 1968. Disponível em: < http://books.google.com.br >. Acesso em: 12, dezembro de 2013.

BORTOLASO, I. V.; BALESTRIN, A. TEIXEIRA; R. FACCIN, K. The Trajectory of Brazilian Semiconductor Industry: An Economic, Governmental, and Technological Perspectives. Porto Alegre: IAMOT, 2013.

BRASIL. MCT/CNPq/CI-Brasil n 59/2008 - Design Houses - Brasília, 2008. 


\section{A VISÃO RELACIONAL DE POLÍTICAS DE CIÊNCIA E TECNOLOGIA: O CASO DO PROGRAMA CI BRASIL}

CHILD, J.; FAULKNER, D.; TALLMAN, S. Cooperative Strategy: Managing Alliances, Networks and Joint Ventures. 2. ed. [s.1.] Oxford Press, 2005.

CI-Brasil. Design Houses. Disponível em: <http://www.ci-brasil.gov.br/index.php/designhouse.html>. Acesso em: 16 dez. 2012.

CROPPER, S.; EBERS, M.; HUXHAM, C.; RING, P.S. Introducing interorganizational relations. In: The oxford handbook of inter-organizational relations. New York: Oxford University Press Inc. (p. 3-30), 2008.

DAGNINO, R.. A relação universidade-empresa no Brasil e o "Argumento da Hélice Tripla". Revista Brasileira de Inovação - RBI, Rio de Janeiro, v. 2, n. 2, p. 267-307, dez. 2003.

DIAS, C. N. Rede de cooperação social como estratégia para a implementação de políticas públicas: O Caso da PNDR. In: V Encontro de Estudos em Estratégia - 3Es. ANPAD Porto Alegre/RS, 2011.

DYER, J. H.; SINGH, H. The relational view: Cooperative strategy and sources of interorganizational. Academy of Management Review, p. 660-679, 1998.

EBERS, M.; JARILLO, Carlos J..The construction, forms, and consequences of industry networks. International Studies of Management e Organization, [s.1.], v. 27, p. 3-21, Winter, 1998.

EDMONDSON, G. et al. Making Industry-University Partnerships Work: Lessons from successful collaborations. Science Business Innovation Board AISBL, p. 1-52, 2012.

EMERSON, R.M. Power-Dependence Relations. American Sociological Review, n.27, p.3140, 1962.

ETZKOWITZ, H. Hélice Tríplice: Universidade - Indústria - Governo Inovação em movimento. Porto Alegre: ediPUCRS, 2009.

ETZKOWITZ, H.; LEYDESDORFF, L. The dynamics of innovation: from national systems "mode 2" to a tripe helix of university-industry-government relations. Research Policy, Amsterdam, v. 29, n. 2, p. 109-123, 2000.

ETZKOWITZ, H.; LEYDESDORFF, L. The triple helix as a model for innovations studies. In: Triple Helix Conference, 2. Purchase, 1998.s/l.: s/n., 1998. (Conference Report).

ETZKOWITZ, H.; LEYDESDORFF, L. Universities in the global economy: a triple helix of University-Industry-government relations. London: Cassel Academic, 1997. 
ETZKOWITZ, H.; LEYDESDORFF, L. The triple helix-university-industry-government relations: a laboratory for knowledge-based economic development. European Association Study Science and Technology Review, London, v. 14, n. 1, p. 14-19, 1995.

FACCIN,K.; BALESTRIN,A.; BORTOLASO, I.V.; The joint R\&D project: The case of the first Brazilian microcontroller chip. R.Adm., São Paulo, v.51, n.1, p.87-102, jan./fev./mar. 2016.

FLEURY, S. OUVERNEY, A.M. Gestão de Redes: a estratégia de regionalização da política de saúde. Rio de Janeiro: FGV, 2007.

FREEMAN, C. The national system of innovation in historical perspective. Cambridge Journal of Economics, v. 9, n. 1, p. 5-24, 1995.

GEDDES, Mike. Inter-organizational Relationships in Local and Regional Development Partnerships. Inter-organizational Relationships, Chains and Networks: A Supply Perspective. In: CROPPER, S. et al. (Orgs). The Oxford Handbook of Inter-organizational Relations. New York: Oxford University Press, 2008.

GUTIERREZ, R.; LEAL, C. Estratégias para uma Indústria de Circuitos Integrados no Brasil. 2004. Disponível em: < http://www.bndes.gov.br >. Acesso em: 16/12/2012.

IBARRA, H.; HANSEN, M. T.. Are you Collaborative Leader. Harvard Business Review, v. 89, n.7-8, p. 68-74, 2011.

KLIJN, E..Policy and Implementation Networks. Inter-organizational Relationships, Chains and Networks: A Supply Perspective. In: CROPPER, S. et al. (Orgs). The Oxford Handbook of Inter-organizational Relations. New York: Oxford University Press, 2008.

KRAAIJENBRINK, J.; SPENDER J.-C.; GROEN, A. J.. The resource-based view: A review and assessment of its critiques. Journal of Management, v. 36, p. 349-372, 2010.

LITWAK, E.; HYLTON, L.F. Interorganizational Analysis: A hypothesis on Coordinating Agencies. Administrative Science Quarterly, p.395-420, 1962.

LÖFSTRÖM, M. Inter-organizational collaboration projects in the public sector: A balance between integration and demarcation. International Journal of Health Planning and Management, v. 25, n. 2, p. 36-55, 2010.

LUNDBERG, H.; ANDRESEN, E. Cooperation among companies, universities and local government in a Swedish context. Industrial Marketing Management, v. 41, n. 3, p. 429437, abr. 2012.

MCTI. Ministério da Ciência, tecnologia e Inovação. CI Brasil: Caderno Informativo. Ministério da Ciência e Tecnologia, Brasília, 2011. 


\section{A VISÃO RELACIONAL DE POLÍTICAS DE CIÊNCIA E TECNOLOGIA: \\ O CASO DO PROGRAMA CI BRASIL}

NALEBUFF, Barry J.; BRANDENBURGER, Adam M. Co-opetição. Rio de Janeiro: Rocco, 1995.

NATÁRIO, M. M.; COUTO, J. P. A.; ALMEIDA, C. F. R ... The triple helix model and dynamics of innovation: a case study. Journal of Knowledge-based Innovation in China, v.4, n. 1, p.36-54, 2012.

PORTER, M.E. Competitive Strategy. New York: Free Press. 1980.

REVISTA AMANHÃ. O vale do Silício. Porto Alegre. p. 38-46, nov. 2012.

RHODES, R.A.W. Beyond Westminter and Whitehall: The Sub-Central Governments of Brain. London: Unwin Hyman. 1988.

SAAD, M.; ZAWDIE, G.; MALAIRAJA, C. The Triple helix strategy for universities in developing countries: the experiences in Malaysia and Algeria. Science and Public Policy, v.35, n.6, p.431-443, 2008.

SANDFORT, J.R.; MILWARD, H.B. Collaborative service provision in the public sector. CROPPER, S. et al. (Orgs). The Oxford Handbook of Inter-organizational Relations. New York: Oxford University Press, 2008.

SIA. Semiconductor Industry Association. Disponível em: <http://www.siaonline.org/news/2014/12/03/global-sales-reports-2014/global-semiconductor-sales-flatmonth-on-month>. Acesso em: 11 jan. 2015.

TODLING, F.; LEHNER, P.; KAUFMANN, A. Do different types of innovation rely on specific kinds of knowledge interactions? Technovation, n. 29, p. 59-71, 2009.

ZAHEER, A.; GOZUBUYUK, R.; MILANOV, H.. It's the Connections: the Network Perspective in Interorganizational Research. Academy of Management Perspectives, v. 24, n. 1, p. 62-77, 2010. 